Original Article

\title{
RESPIRATORY MUSCLE TRAINING WITH THERABAND VERSUS INCENTIVE SPIROMETER AMONG INDIVIDUALS WITH BRONCHIAL ASTHMA
}

\author{
K. REKHA1 ${ }^{1}$ D. SAMUEL SUNDAR DOSS ${ }^{*}{ }^{*}$ MOHAMMED ABUBAKKER SIDDIUE²
}

${ }^{1}$ Saveetha College of Physiotherapy, Chennai, India, ${ }^{* 1}$ Tutor, Saveetha Medical College and Hospital, Chennai, India, ${ }^{2 B P T}$ Student, Saveetha College of Physiotherapy, Chennai, India

Email: samsundar09@gmail.com

Received: 20 Jan 2020, Revised and Accepted: 19 Mar 2020

\section{ABSTRACT}

Objective: To determine and compare the effects of Theraband exercises with Incentive Spirometer exercise in improving respiratory muscle strength among bronchial asthma.

Methods: In this study, 30 Individuals with Bronchial asthma were taken and assessed for FEV1/FVC, Maximum voluntary ventilation (MVV) using a computerized spirometer, dyspnea level graded using MMRC scale and diaphragm muscle strength assessed using manual muscle testing (MMT) as pretest then the individuals were randomly allocated in two groups: Group A and Group B. Group A ( $\mathrm{n}=15)$ received Theraband exercise and Group B (n=15) were treated with incentive spirometer for a total period of $4 \mathrm{w}$ following which the outcome measures were repeated for post-test.

Results: Based on the obtained statistics, both the groups showed significant improvement within groups when comparing pre and post-test measures. On comparing the mean values of the post-test between the groups, theraband exercises showed clinically higher significant results than incentive spirometer exercises.

Conclusion: From the result, it shall been concluded that both theraband exercises and incentive spirometer exercises shows improvement in MVV, FEV1/FVC, reduced dyspnea and increased respiratory muscle strength. Hence, theraband exercises are effective compared to the incentive spirometer.

Keywords: Theraband Versus, Bronchial Asthma

(C) 2020 The Authors. Published by Innovare Academic Sciences Pvt Ltd. This is an open access article under the CC BY license (http://creativecommons.org/licenses/by/4.0/)

DOI: http://dx.doi.org/10.22159/ijcpr.2020v12i3.38319. Journal homepage: https://innovareacademics.in/journals/index.php/ijcpr

\section{INTRODUCTION}

Bronchial asthma is an atopic disease, it affects around $7 \%$ of the population, and 300 million people are affected worldwide. The disease is characterized by chronic airway inflammation and increased airway hyperresponsiveness which presents the symptoms of wheeze, cough and dyspnea. It is known that the prevalence of Asthma is increasing steadily in recent years, especially in countries with a western lifestyle and also in developing countries [1].

In India the estimated prevalence rate is about $3 \%$ which is nearly 30 million patients consists of $2.4 \%$ adults and $4 \%-20 \%$ children's [2] The patients who under-estimate their symptoms are under high risk. About $15-20 \%$ of asthmatics individuals are unnoticed of moderate changes in their airflow obstruction and which may quickly deteriorate until they suddenly present with severe asthma [3].

Asthmatic patients are exposed to the high resistance of their airways and also hyperinflation of their lungs, which leads to a shortening of their inspiratory muscles and also flattens the diaphragm and places them at a mechanical disadvantage. In addition to that, a large amount of pressure work are required by the inspiratory muscles to overcome high airway resistance [4].

The Non-medical therapy of asthma consists of various aspects of patient's guidance including education and also various forms of physiotherapy. The most common interventions for strengthening of respiratory muscles among physiotherapist include breathing exercises, thoracic mobility exercises and also strengthening done through various gadgets and devices, which include Swiss ball, Positive expiratory pressure device, incentive spirometer etc. The ultimate aim of the intervention is to reduce respiratory symptoms. Since respiratory muscles are weekend in Bronchial Asthma, strengthening respiratory muscles is more important. Strengthening can be done by various means and in this study, strengthening is given by Incentive spirometer and theraband [5].

Incentive spirometer is a portable, inexpensive device which is used clinically as a part of the routine prophylactic and therapeutic regimen.
Since the first incentive spirometer was constructed by Bartlett et al. in the 1970's, consists of a cylinder and piston with an adjustable volume of $2000-2500 \mathrm{ml}$, this type of incentive spirometer is used as an alternative to IPPB for several years [6]. Bartlett-Edwards incentive spirometer remained standard for many years, later many different types of incentive spirometers had been developed, which are less expensive and also single-use units [7]. It is accomplished by the providence of visual feedback; that is, breathing is visualized by an uplifted plate or ball in a transparent cylinder during sustained inspiration, which is activated by an inspiratory effort [8].

The Theraband was made in Akron, USA in 1978. These elastic bands are safe, inexpensive and portable, which are highly versatile tools that are often used in therapeutic exercise programs and multipurpose physical training activities. Its unique properties are that it allows muscles to be stretched and relaxed in a smooth and consistent manner. Their physical characteristic is based on stress-strain relationships, the more the band is stretched; the greater the resistance to further elongation. This also prevents the bounce at the end of a range of motion exercise that can cause muscle spasms. The other major advantage is that it is independent on gravity for providing resistance $[9,10]$.

These bands are available in various thickness and resistance depending on its varying colour's. Tan and yellow band offers minimal resistance, red, green, blue and black bands offers medium resistance where else heavy silver and gold colour offers maximal resistance [11]. Though these bands are endorsed even by American Physical Therapy Association (APTA), it is not commonly used in cardio-respiratory impairments and not seems to be used in bronchial asthma. So this study introduces the application of Theraband exercises in the strengthening of respiratory muscles in bronchial asthma individuals. Thereby in this study, we intend to analyze the effects of Theraband and Incentive spirometer exercises in training the Respiratory muscle among individuals with bronchial asthma.

\section{MATERIALS AND METHODS}

A Quasi-experimental study was conducted at Saveetha medical college hospital. Scientific review board approval and ethical committee 
approval was obtained prior to the study. 30 bronchial asthma patients were selected based on inclusion and exclusion criteria. A Consecutive sampling technique used to allocate the participants equally into two groups Group A and Group B by the Odd-Even method.

Study included Males and females with Bronchial Asthma (Mildmoderate persistence type), those individuals under Stable medical treatment (no changes $<4 \mathrm{w}$ before the start of baseline testing), Age group-25 to $55 \mathrm{y}$ and study excluded those with status asthmaticus, recent thoracic surgeries, recent trauma, unstable vital signs, systemic diseases affecting muscles and joints, any neurological disorders, significant co-morbidity including malignancy, and symptomatic cardiovascular disease.

Detailed procedure was explained to the patient in their native language with an information sheet and those who are interested, were included in the study after obtaining a written informed consent from all the participants. After which all the participants assessed for pre test outcomes, Forced Expiratory Volume in one second/forced vital capacity (FEV1/FVC), Maximum voluntary ventilation (MVV) using a computerized spirometer, dyspnea level graded using Medical Research Council (MMRC) scale and diaphragm muscle strength assessed using manual muscle testing (MMT) as pre-test. Group A $(n=15)$ received theraband exercises and Group B $(n=15)$ was treated with an incentive spirometer for a total period of $4 \mathrm{w}$ following which the pre-test outcome measures were repeated for post-test.

Group A treated with Theraband exercises, all the patients were treated with low resistance bands (yellow colour).

\section{Exercise 1: Reverse flies}

- Patient is asked to stand in a comfortable position.

- Then patient is asked to hold the ends of elastic band in his both hands forwards with shoulder flexed to 90 degree with his elbows straight.

- Now the patient is asked to abduct his both hands up to 90 degree, simultaneously pulling the bands outwards.

- Then slowly return to the start position.

- Repeat the exercise

\section{Exercise 2: Dynamic hug}

- Patient is asked to stand in a comfortable position.

- Patient is asked to wind up the theraband around his chest wall at

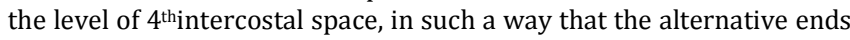
of the band is in opposite hands of the patient.

- Then the patient is asked to breath-in while pulling the ends of the band on both sides simultaneously and breathe-out in relaxing the band.

- Repeat the exercise

\section{Exercise 3: chest flies}

- Patient is positioned in front of the window, in walk stance position with the forward knee mid flexed. And patient back facing the window.

- Middle of the elastic band is fixed to the window at shoulder level.

- Patient is then asked to grasp elastic band in his both hands at shoulder level with his elbows straight pulling from the band from abduction to flexion forwards.
- Then slowly return to the start position.

- Repeat the exercise

\section{Exercise 4 (chest press)}

- Patient is asked to stand comfortably

- Middle of the elastic band is fixed to the window at shoulder level.

- Patient is then asked to hold elastic band in his both hands at shoulder level with his elbows flexed in shoulder abduction.

- Patient is then asked to pull the band forward by straightening his elbows and flexing the shoulder.

- Then slowly return to the start position.

- Repeat the exercise.

Exercise protocol-Frequency for all the exercises are performed for

- 8-10 repetitions/set,

- Each exercise Hold time: 10 seconds; Rest time: 15 seconds

- $4 \mathrm{~d} /$ week

Group B: $(n=15)$ is treated with Incentive spirometer device.

- Patient was asked to sit in a well-supported chair or couch comfortably,

- Patient is asked to place the mouthpiece of the device in his mouth with lips sealed around the mouthpiece and then the patient is asked to inhale slowly and deeply throughout the procedure

- Patient is asked to watch the incentive spirometer to raise the indicator, and the patient is instructed to sustain the indicator at the end of inspiration for at least 2 seconds.

- Then patient is asked to exhale normally.

- 10 repetitions/set-5 sets with 2 min interval between each set.

- Repeat the procedure for $20 \mathrm{~min}$

- Frequency: $4 \mathrm{~d} /$ week

- Duration: $4 \mathrm{w}$

Both the group patients were asked to continue the medications prescribed by the physician if any, during the intervention period

\section{RESULTS}

Statistical analysis was carried out for the collected data using descriptive and inferential statistics. Mean and the standard deviation was calculated for different parameters in pre test and post test. The mean value and standard errors were calculated for different variables and the difference in mean value was tested for statistical significance using paired $\mathrm{t}$ test. $\mathrm{P}$ value $<0.05$ was considered as statistically significant. Paired t-test was used to analyze significant changes between pre-test and post-test measurements within the group. Unpaired t-test was used to analyze significant changes between two groups.

Statistical analysis of post-test values of FEV1/FVC, MVV, MMT grades and MMRC dyspnea scores revealed that there is a statistically significant difference seen between Group-A and Group-B.

Table 1: Comparison of FEV1/FVC for Group A and B

\begin{tabular}{llllll}
\hline & Mean & & \multicolumn{2}{l}{ Standard deviation } & \\
\hline & Pre-test & Post-test & Pre-test & Post test & t-value \\
\hline Group A & 69.20 & 80.80 & 3.30 & 8.50 & 6.148 \\
Group B & 69.20 & 74.33 & 3.30 & 4.92 & 10.148 \\
\hline
\end{tabular}


Table 2: Comparison of MVV for group A and B

\begin{tabular}{|c|c|c|c|c|c|c|}
\hline & \multicolumn{2}{|l|}{ Mean } & \multicolumn{4}{|c|}{ Standard deviation } \\
\hline & Pre-test & Pre-test & Pre-test & Post test & t-value & p-value \\
\hline Group A & 83.67 & 103.07 & 12.12 & 12.14 & 8.8478 & 0.0001 \\
\hline Group B & 69.59 & 89.53 & 10.13 & 8.78 & 7.658 & 0.0001 \\
\hline
\end{tabular}

Table 3: Manual muscle testing (MMT) for the diaphragm to group A and B

\begin{tabular}{llllll}
\hline & Mean & & \multicolumn{3}{l}{ Standard deviation } \\
\hline & Pre-test & Post-test & Pre-test & Post test & t-value \\
\hline Group A & 2.33 & 3.60 & 0.98 & 0.99 & 10.7174 \\
Group B & 2.33 & 2.73 & 0.98 & 0.88 & 0.001 \\
\hline
\end{tabular}

Table 4: MMRC dyspnea scale values of group-A and group-B

\begin{tabular}{llllll}
\hline & Mean & & \multicolumn{2}{l}{ Standard deviation } \\
\hline & Pre-test & Post-test & Pre-test & Post test & t-value \\
\hline GROUP A & 2.07 & 0.80 & 0.80 & 0.68 & 10.7174 \\
GROUP B & 1.93 & 1.47 & 0.80 & 0.92 & 3.5000 \\
\hline
\end{tabular}

\section{DISCUSSION}

Asthma has an influence on lung volumes and capacities, it is known that strength and endurance of respiratory muscles is affected among asthma patients, which has an impact on patient's quality of life, as the symptoms of asthma such as dyspnea, wheeze drastically reduces the functional activities of the individual, limiting their activities which affects their lifestyle. For these reasons Asthma patients need to be treated with both medical and nonmedical therapy. So the aim of the treatment is to achieve Normal functional activities with increased exercise capacity, in order to prevent recurrent asthma attacks and to achieve optimal lung functions with or without minimal symptoms [5].

Weiner et al., has demonstrated that asthma patients are exposed to increase airway resistance and hyperinflation. Airway resistance increased up to 15 times normal and also the probably the concomitant hyperinflation impairs the capacity of the respiratory muscles to handle this increased load. A number of studies have been carried out to correlate dyspnea and respiratory muscle performance. It was well documented that the intensity of breathlessness is related to the activity and the strength of the inspiratory muscles. For these reasons in our study we determine to strengthen respiratory muscles among asthma patients. It has been observed statistical from the results that symptoms of dyspnea is reduces, and increased respiratory muscle strength by increasing MVV, FEV1/FVC and MMT score of diaphragm.

It has been shown on previous study on bronchial asthma, that inspiratory muscle training with threshold device and breathing exercises were effective to promote biomechanical factors of respiratory muscle functions during expiration and inspiration [12]. There are numerous studies on the therapeutic and physiological efficacy of incentive spirometer on respiratory care; clinical evidences also co-relates its effects on pulmonary functions and increased respiratory muscle strength.

In this study, we also introduce the theraband exercise techniques, for the first time to bronchial asthma patients, and thereby minimize its symptoms. We trained mild to moderate asthma patients with specific theraband exercise training and compare them with standard incentive spirometer exercise training, which is proven to be significant in both clinically and statistically.

\section{CONCLUSION}

The theraband exercises and incentive spirometer exercises shows clinical benefits in bronchial asthma individuals. From the result, it shall been concluded that both the Theraband exercises and incentive spirometer exercises shows improvement in pulmonary functions of MVV and FEV1/FVC, reduces dyspnea and increases the respiratory muscle strength. Whereas, the theraband exercises are proven to be effective than routinely used incentive spirometer exercise training.

\section{FUNDING}

NIL

\section{AUTHORS CONTRIBUTIONS}

All the Authors contributed equally.

\section{CONFLICT OF INTERESTS}

None

\section{REFERENCES}

1. Halim T, Alam MY Ali, MMSU Islam, F Ahammed, SH Rahman, RC Barman. A prospective study of bronchial asthma. Faridpur Med Coll J 2011;6:100-3.

2. Agarwal S, Pearce N, S Ebrahim. Prevalance and risk factors of self-reported asthma in Indian population. Int J Tuberc Lung Disease 2013;17:275-82.

3. Butter worth, Heinemann. Tidy"sphysiotherapy edited by Stuart B. Porter. $13^{\text {th }}$ edition. Elsevier Science Limited; 2003. p. 303.

4. Paltiel Weiner, Yaizazgad, Rasemganan, Margalit Weiner. Inspiratory muscles training in patients with bronchial asthma. Chest 1992;102:1357-61.

5. Marjolein LJ Bruus, lianne J, Vander Giessen, Heleenmoed. The effectiveness of physiotherapy in patients with asthma: a systemic review of the literature. J Respir Med 2013;107:483-98.

6. GD Gale, DE Sanders. The barlett-edwards incentive spirometer: a preliminary assessment of its use in the prevention of the atelectasis after cardiopulmonary bypass. Can Anaesth Soc J 1977;24:408-16.

7. Tom J Overand, Catherine M Anderson, S Deborahlucy, Christina Bhatia, Birgitta I. Jonsson, Catherine Timmermans. The effects of incentive spirometry on postoperative pulmonary complications. Chest 2001;120:971-8.

8. Ruben D Restrepo, Richard Wettstein, Leowittnebel, Michael Trancy. AARC clinical practice guideline of Incentive spirometry. Respir Care 2011;56:1600-4.

9. Marco Uchida, Marco Makoto Nishida, Ricardo Aurelio Carval Hosampio, Toshio Moritani, Hidenori Aria. Theraband-elastic band tension: reference value for physical activity. J Phys Ther Sci 2016;28:1266-71.

10. Guy G Simoneau, Shellie M Bereda, Dennis C Sobush, Andrew J Satarsky. Biomechanics of elastic resistance in therapeutic exercise programs. J Orthopaedic Sports Phys Thera 2001;31:1624.

11. https://www.bindependent.com/hompg/bi/bindep/store/aisles /exercise/bands/Theraband.htm [Last accessed on 10 Dec 2019]

12. Shiny S James, K Rekha, Vaipyapuri Anandh. Effects of threshold inspiratory muscle trainer in bronchial asthma. Biomed J 2016;36:77-82.

13. N Aggarwal, K Chaudhry, SK Chhabra, GA D'Souza, D Gupta, SK Jindal, SK Katiyar, et al. Prevalence and risk factors for bronchial 
asthma in Indian adults: a multicentre study. Indian J Chest Diseases Allied Sci 2006;48:13-22.

14. Turner LA, Mickleborough TD, McConnell AK, Stager JM, Tecklenburg Lund S, Lindley MR. Effect of inspiratory muscle training on exercise tolerance in asthmatic individuals. Med Sci Sports Exercise 2011;43:2031-8.

15. Andre Nyberg, Didier Saey, Mickaël Martin, François Maltais Muscular and functional effects of partitioning exercising muscle mass in patients with chronic obstructive pulmonary disease-a study protocol for a randomized controlled trial. Trials 2015;16:194.

16. Lawrence $P$ Cahalin, Ross Arena. Impact of exercise training on adverse event risk and quality of life in patients with heart failure. Phys Ther 2012;92:1371-5.

17. Lars L Andersen, Christoffer H Andersen, Ole S Mortensen, Otto M Poulsen, Inger Birthe $\mathrm{T}$ Bjornlund, Mette $\mathrm{K}$ Zebis. Muscle activation and perceived loading during rehabilitation exercises: comparison of dumbbells and elastic resistance. Phys Thera 2010;90:538-49.

18. Phillip A Page, John Lamberth, Ben Abadie, Robert Boling, Robert Collins, Russell Linton. Posterior rotator cuff strengthening using

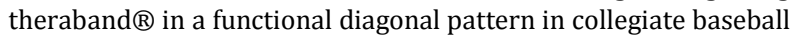
pitchers. J Athletic Training 1993;28:346-54.

19. Sanjeev Kumar Khanna. Efficacy of incentive spirometer in improving pulmonary functions after upper abdominal surgery. Indian J Basic Appl Med Res 2013;3:328-34.

20. Harpreet Ranu, Michael Wilde, Brendan Madden. Pulmonary function tests. Ulster Med J 2011;80:84-90.

21. Donald A Mahler, Carolyn K Wells. Evaluation of clinical methods for rating Dyspnea. Chest J 1988;93:580-6. 